Legendre curves i $n$ the unit spherical bundl e over the unit sphere and evol utes

\begin{tabular}{|l|l|}
\hline 著者 & TAKAHASH Nasat ond \\
\hline $\begin{array}{l}\text { j our nal or } \\
\text { publ i cat } \mathrm{i} \text { on } \mathrm{t} \text { i t l e }\end{array}$ & Cont empor ar y Nat hemat i cs \\
\hline vol une & 675 \\
\hline page $\mathrm{r}$ ange & $337-355$ \\
\hline year & 2016 \\
\hline URL & ht t p: //hdl . handl e. net $/ 10258 / 00009568$ \\
\hline
\end{tabular}




\title{
Legendre curves in the unit spherical bundle over the unit sphere and evolutes
}

\author{
Masatomo Takahashi * \\ Dedicated to Professor Maria del Carmen Romero Fuster \\ on the occasion of her 60th birthday
}

September 16, 2015

\begin{abstract}
In order to consider singular curves in the unit sphere, we consider Legendre curves in the unit spherical bundle over the unit sphere. By using a moving frame, we define the curvature of Legendre curves in the unit spherical bundle. As applications, we give a relationship among Legendre curves in the unit spherical bundle, Legendre curves in the unit tangent bundle and framed curves in the Euclidean space, respectively. Moreover, we define not only an evolute of a front, but also an evolute of a frontal in the unit sphere under certain conditions. Since the evolute of a front is also a front, we can take evolute again. On the other hand, the evolute of a frontal if exists, is also a frontal. We give an existence and uniqueness conditions of the evolute of a frontal.
\end{abstract}

\section{Introduction}

For regular curves in the unit sphere, the Frenet Serret formula and the geodesic curvature are important to investigate geometric properties of the regular curves. On the other hand, for singular curves in the unit sphere, we can not construct the Frenet Serret formula and the geodesic curvature at singular points of the curve. For singular curves, V. I. Arnold established the spherical geometry by using Legendre singularity theory [2]. It studied fronts in the unit sphere and gave properties of fronts. Some results in this paper have already considered in $[2,16,17,20,21]$. However, we clarify the notations and calculations by using the curvature of Legendre curves in the unit spherical bundle over the unit sphere. By using the curvature of the Legendre curves, we give existence and uniqueness theorems of Legendre curves in the unit spherical bundle in $\S 2$. We also give relationships among Legendre curves in the unit spherical bundle, Legendre curves in the unit tangent bundle and framed curves in the Euclidean space,

\footnotetext{
*Supported by JSPS KAKENHI Grant Number No.26400078.

2010 Mathematics Subject classification: 58K05, 53A40, 53D35.

Key Words and Phrases. spherical Legendre curve, frontal, front, curvature, evolute.
} 
respectively in $\S 3$. Moreover, we define not only an evolute of a front in $\S 4$, but also an evolute of a frontal in the unit sphere under certain conditions in $§ 5$. Since the evolute of a front is also a front, we can take evolute again. We give $k$-th evolute of the front and its curvature inductively. On the other hand, the evolute of a frontal if exists, is also a frontal. We give an existence and uniqueness conditions of the evolute of a frontal. It is a quit different property between the evolute of a frontal in the sphere and in the Euclidean plane (cf. [8]). We also give examples of evolutes of a front and a frontal in $\S 6$.

All maps and manifolds considered here are differentiable of class $C^{\infty}$.

Acknowledgement. I would like to thank the referee for helpful comments to improve the original manuscript.

\section{Legendre curves in the unit spherical bundle}

Let $\mathbb{R}^{3}$ be the 3 -dimensional Euclidean space. The inner product on $\mathbb{R}^{3}$ is given by $\boldsymbol{a} \cdot \boldsymbol{b}=$ $a_{1} b_{1}+a_{2} b_{2}+a_{3} b_{3}$ and the vector product of $\boldsymbol{a}$ and $\boldsymbol{b}$ on $\mathbb{R}^{3}$ is given by

$$
\boldsymbol{a} \times \boldsymbol{b}=\left|\begin{array}{ccc}
\boldsymbol{e}_{1} & \boldsymbol{e}_{2} & \boldsymbol{e}_{3} \\
a_{1} & a_{2} & a_{3} \\
b_{1} & b_{2} & b_{3}
\end{array}\right|
$$

where $\boldsymbol{e}_{1}, \boldsymbol{e}_{2}, \boldsymbol{e}_{3}$ is the canonical basis on $\mathbb{R}^{3}, \boldsymbol{a}=\left(a_{1}, a_{2}, a_{3}\right)$ and $\boldsymbol{b}=\left(b_{1}, b_{2}, b_{3}\right)$. We denote the unit sphere $S^{2}=\left\{\boldsymbol{x} \in \mathbb{R}^{3} \mid \boldsymbol{x} \cdot \boldsymbol{x}=1\right\}$.

Let $\gamma: I \rightarrow S^{2}$ be a regular curve. We define the unit tangent vector $\boldsymbol{t}(t)=\dot{\gamma}(t) /|\dot{\gamma}(t)|$ and the unit normal vector $\boldsymbol{n}(t)=\gamma(t) \times \dot{\gamma}(t) /|\dot{\gamma}(t)|$, where $|\dot{\gamma}(t)|=\sqrt{\dot{\gamma}(t) \cdot \dot{\gamma}(t)}$ and $\dot{\gamma}(t)=$ $(d \gamma / d t)(t)$. Then $\{\gamma(t), \boldsymbol{t}(t), \boldsymbol{n}(t)\}$ is a moving frame along $\gamma(t)$ and the Frenet Serret formula is given by

$$
\left(\begin{array}{c}
\dot{\gamma}(t) \\
\dot{\boldsymbol{t}}(t) \\
\dot{\boldsymbol{n}}(t)
\end{array}\right)=\left(\begin{array}{ccc}
0 & |\dot{\gamma}(t)| & 0 \\
-|\dot{\gamma}(t)| & 0 & |\dot{\gamma}(t)| \kappa_{g}(t) \\
0 & -|\dot{\gamma}(t)| \kappa_{g}(t) & 0
\end{array}\right)\left(\begin{array}{c}
\gamma(t) \\
\boldsymbol{t}(t) \\
\boldsymbol{n}(t)
\end{array}\right)
$$

where the geodesic curvature $\kappa_{g}$ is given by

$$
\kappa_{g}(t)=\frac{\dot{\boldsymbol{t}}(t) \cdot \boldsymbol{n}(t)}{|\dot{\gamma}(t)|}=\frac{\operatorname{det}(\gamma(t), \dot{\gamma}(t), \ddot{\gamma}(t))}{|\dot{\gamma}(t)|^{3}} .
$$

The evolute $E v(\gamma): I \rightarrow S^{2}$ of a regular curve $\gamma: I \rightarrow S^{2}$ is given by

$$
E v(\gamma)(t)= \pm \frac{\kappa_{g}(t)}{\sqrt{\kappa_{g}^{2}(t)+1}} \gamma(t) \pm \frac{1}{\sqrt{\kappa_{g}^{2}(t)+1}} \boldsymbol{n}(t)
$$

By definition, we can not construct the Frenet Serret formula at singular points of $\gamma: I \rightarrow$ $S^{2}$. In this paper, we would like to consider singular curves in the unit sphere.

We denote $\Delta=\left\{(\boldsymbol{a}, \boldsymbol{b}) \in S^{2} \times S^{2} \mid \boldsymbol{a} \cdot \boldsymbol{b}=0\right\}$ and is a 3-dimensional manifold .

Definition 2.1 We say that $(\gamma, \nu): I \rightarrow \Delta \subset S^{2} \times S^{2}$ is a Legendre curve (or, spherical Legendre curve) if $\dot{\gamma}(t) \cdot \nu(t)=0$ for all $t \in I$. We call $\gamma$ a frontal and $\nu$ a dual of $\gamma$. Moreover, if $(\gamma, \nu)$ is an immersion, we call $\gamma$ a front. 
We consider the canonical contact structure on the unit spherical bundle $T_{1} S^{2}=S^{2} \times S^{2}$ over $S^{2}$. If $(\gamma, \nu)$ is a Legendre curve, then $(\gamma, \nu)$ is an integral curve with respect to the contact structure (cf. [2]).

We define $\boldsymbol{\mu}(t)=\gamma(t) \times \nu(t)$. Then $\boldsymbol{\mu}(t) \in S^{2}, \gamma(t) \cdot \boldsymbol{\mu}(t)=0$ and $\nu(t) \cdot \boldsymbol{\mu}(t)=0$. It follows that $\{\gamma(t), \nu(t), \boldsymbol{\mu}(t)\}$ is a moving frame along the frontal $\gamma(t)$. By the standard arguments, we have the Frenet Serret type formula as follows:

Proposition 2.2 Let $(\gamma, \nu): I \rightarrow \Delta$ be a Legendre curve. Then we have

$$
\left(\begin{array}{c}
\dot{\gamma}(t) \\
\dot{\nu}(t) \\
\dot{\boldsymbol{\mu}}(t)
\end{array}\right)=\left(\begin{array}{ccc}
0 & 0 & m(t) \\
0 & 0 & n(t) \\
-m(t) & -n(t) & 0
\end{array}\right)\left(\begin{array}{c}
\gamma(t) \\
\nu(t) \\
\boldsymbol{\mu}(t)
\end{array}\right)
$$

where $m(t)=\dot{\gamma}(t) \cdot \boldsymbol{\mu}(t)$ and $n(t)=\dot{\nu}(t) \cdot \boldsymbol{\mu}(t)$.

We say that the pair of the functions $(m, n)$ is the curvature of the Legendre curve $(\gamma, \nu): I \rightarrow$ $\Delta \subset S^{2} \times S^{2}$.

Note that $t_{0}$ is a singular point of $\gamma$ (respectively, $\nu$ ) if and only if $m\left(t_{0}\right)=0$ (respectively, $\left.n\left(t_{0}\right)=0\right)$.

Remark 2.3 If $(\gamma, \nu): I \rightarrow \Delta \subset S^{2} \times S^{2}$ is a Legendre curve with the curvature $(m, n)$, then $(\gamma,-\nu)$ is a Legendre curve with the curvature $(-m, n)$. Also $(-\gamma, \nu)$ is a Legendre curve with the curvature $(m,-n)$. Moreover, $(\nu, \gamma)$ is a Legendre curve with the curvature $(-n,-m)$.

Definition 2.4 Let $(\gamma, \nu),(\widetilde{\gamma}, \widetilde{\nu}): I \rightarrow \Delta \subset S^{2} \times S^{2}$ be Legendre curves. We say that $(\gamma, \nu)$ and $(\widetilde{\gamma}, \widetilde{\nu})$ are congruent as Legendre curves if there exists a special orthogonal matrix $A \in S O(3)$ such that

$$
\widetilde{\gamma}(t)=A(\gamma(t)), \widetilde{\nu}(t)=A(\nu(t))
$$

for all $t \in I$.

Then we have the following existence and uniqueness theorems in terms of the curvature of the Legendre curve.

Theorem 2.5 (The Existence Theorem) Let $(m, n): I \rightarrow \mathbb{R} \times \mathbb{R}$ be a smooth mapping. There exists a Legendre curve $(\gamma, \nu): I \rightarrow \Delta \subset S^{2} \times S^{2}$ whose associated curvature is $(m, n)$.

Theorem 2.6 (The Uniqueness Theorem) Let $(\gamma, \nu)$ and $(\widetilde{\gamma}, \widetilde{\nu}): I \rightarrow \Delta \subset S^{2} \times S^{2}$ be Legendre curves whose curvatures $(m, n)$ and $(\widetilde{m}, \widetilde{n})$ coincide. Then $(\gamma, \nu)$ and $(\widetilde{\gamma}, \widetilde{\nu})$ are congruent as Legendre curves.

By using the theorems of the existence and uniqueness of the solution of a system of linear ordinary differential equations, these proofs are similar to the cases of regular space curves ([11]), Legendre curves in the unit tangent bundle $([6])$ and framed curves $([12])$, we omit it.

Example 2.7 Let $\gamma: I \rightarrow S^{2}$ be a regular curve. We consider a Legendre immersion $(\gamma, \boldsymbol{n})$ : $I \rightarrow \Delta \subset S^{2} \times S^{2}$. Then the relationship between the geodesic curvature $\kappa_{g}$ of $\gamma$ and the curvature $(m, n)$ of $(\gamma, \boldsymbol{n})$ is given by $\kappa_{g}(t)=n(t) /|m(t)|$. 
Example 2.8 Let $n, m$ and $k$ be natural numbers with $m=k+n$. We give a mapping $(\gamma, \nu): \mathbb{R} \rightarrow \Delta \subset S^{2} \times S^{2}$ by

$$
\gamma(t)=\frac{1}{\sqrt{1+t^{2 n}+t^{2 m}}}\left(1, t^{n}, t^{m}\right), \nu(t)=\frac{1}{\sqrt{n^{2}+m^{2} t^{2 k}+k^{2} t^{2 m}}}\left(k t^{m},-m t^{k}, n\right) .
$$

Then $(\gamma, \nu)$ is a Legendre curve. By definition, we have

$$
\boldsymbol{\mu}(t)=\frac{1}{\sqrt{\left(1+t^{2 n}+t^{2 m}\right)\left(n^{2}+m^{2} t^{2 k}+k^{2} t^{2 m}\right)}}\left(n t^{n}+m t^{m+k},-n+k t^{2 m},-m t^{k}-k t^{m+n}\right)
$$

and the curvature

$$
m(t)=\frac{-t^{n-1} \sqrt{n^{2}+m^{2} t^{2 k}+k^{2} t^{2 m}}}{1+t^{2 n}+t^{2 m}}, n(t)=\frac{k n m t^{k-1} \sqrt{1+t^{2 n}+t^{2 m}}}{n^{2}+m^{2} t^{2 k}+k^{2} t^{2 m}} .
$$

Let $\gamma:\left(I, t_{0}\right) \rightarrow S^{2}$ be a smooth curve germ and denote $\gamma(t)=(x(t), y(t), z(t))$. It can be shown that, if either $x(t), y(t)$ or $z(t)$ does not belong to $\mathfrak{m}_{1}^{\infty}$, then $\gamma$ is a frontal. Here $\mathfrak{m}_{1}^{\infty}$ is the ideal of infinitely flat function germs (cf. [9]).

Without loss of generality, we suppose that $x(t)$ does not belong to $\mathfrak{m}_{1}^{\infty}$ such that

$$
\text { order } x(t) \leq \text { order } y(t) \leq \text { order } z(t)
$$

Assume that $x\left(t_{0}\right)>0$. By the assumptions and $\gamma(t) \in S^{2}$, there exist smooth function germs $a(t), b(t), c(t)$ around $t_{0}$ such that $y(t)=a(t) x(t), z(t)=b(t) x(t)$ and $\dot{b}(t)=c(t) \dot{a}(t)$. It follows that $\gamma$ is given by

$$
\gamma(t)=\frac{1}{\sqrt{1+a^{2}(t)+b^{2}(t)}}(1, a(t), b(t))
$$

If we take

$$
\nu(t)=\frac{1}{\sqrt{(a(t) c(t)-b(t))^{2}+c^{2}(t)+1}}(a(t) c(t)-b(t),-c(t), 1),
$$

then $(\gamma, \nu)$ is a Legendre curve.

On the other hand, constant maps in $S^{2}$ are also frontal, which do not satisfy the above sufficient condition. In particular an analytic curve germ is always frontal, because if it is infinitely flat, then it is constant.

Let $I$ and $\widetilde{I}$ be intervals. A smooth function $u: \widetilde{I} \rightarrow I$ is a (positive) change of parameter when $u$ is surjective and has a positive derivative at every point. It follows that $u$ is a diffeomorphism.

Let $(\gamma, \nu): I \rightarrow \Delta$ and $(\widetilde{\gamma}, \widetilde{\nu}): \widetilde{I} \rightarrow \Delta$ be Legendre curves whose curvatures are $(m, n)$ and $(\widetilde{m}, \widetilde{n})$ respectively. Suppose that $(\gamma, \nu)$ and $(\widetilde{\gamma}, \widetilde{\nu})$ are parametrically equivalent via the change of parameter $t: \widetilde{I} \rightarrow I$, that is, $(\widetilde{\gamma}(u), \widetilde{\nu}(u))=(\gamma(t(u)), \nu(t(u)))$ for all $u \in \widetilde{I}$. By differentiation, we have

$$
\widetilde{m}(u)=m(t(u)) \dot{t}(u), \widetilde{n}(u)=n(t(u)) \dot{t}(u) .
$$

Hence the curvature is dependent of the parametrization. 
Let $(\gamma, \nu): I \rightarrow \Delta \subset S^{2} \times S^{2}$ be a Legendre curve with the curvature $(m, n)$. We define a parallel curve $\gamma_{\theta}: I \rightarrow S^{2}$ by

$$
\gamma_{\theta}(t)=\cos \theta \gamma(t)+\sin \theta \nu(t)
$$

where $\theta \in[0,2 \pi)$. Then $\gamma_{\theta}$ is a frontal. More precisely, we have the following. We denote $\nu_{\theta}: I \rightarrow S^{2}$ by $\nu_{\theta}(t)=-\sin \theta \gamma(t)+\cos \theta \nu(t)$.

Proposition 2.9 Under the above notations, $\left(\gamma_{\theta}, \nu_{\theta}\right): I \rightarrow \Delta \subset S^{2} \times S^{2}$ is a Legendre curve with the curvature

$$
(m(t) \cos \theta+n(t) \sin \theta,-m(t) \sin \theta+n(t) \cos \theta) .
$$

Proof. By definition, $\gamma_{\theta}(t) \cdot \nu_{\theta}(t)=0$. Since $\dot{\gamma}_{\theta}(t)=(m(t) \cos \theta+n(t) \sin \theta) \boldsymbol{\mu}(t)$, then $\dot{\gamma}_{\theta}(t)$. $\nu_{\theta}(t)=0$. It follows that $\left(\gamma_{\theta}, \nu_{\theta}\right)$ is a Legendre curve. Moreover, we have $\boldsymbol{\mu}_{\theta}(t)=\gamma_{\theta}(t) \times \nu_{\theta}(t)=$ $\boldsymbol{\mu}(t)$ and $\dot{\nu}_{\theta}(t)=(-m(t) \sin \theta+n(t) \cos \theta) \boldsymbol{\mu}(t)$. The curvature of the Legendre curve is given by $(m(t) \cos \theta+n(t) \sin \theta,-m(t) \sin \theta+n(t) \cos \theta)$.

We say that $\left(\gamma_{\theta}, \nu_{\theta}\right)$ is a parallel Legendre curve of the Legendre curve $(\gamma, \nu)$. Note that if $(\gamma, \nu)$ is a Legendre immersion, then $\left(\gamma_{\theta}, \nu_{\theta}\right)$ is also a Legendre immersion.

\section{Relationships among spherical Legendre curves, Leg- endre curves and framed curves}

First, we give a relationship between spherical Legendre curves and Legendre curves in the unit tangent bundle over $\mathbb{R}^{2}$.

We review on the Legendre curves in the unit tangent bundle over $\mathbb{R}^{2}$, for more detail see [6]. We say that $(\gamma, \nu): I \rightarrow \mathbb{R}^{2} \times S^{1}$ is a Legendre curve if $(\gamma(t), \nu(t))^{*} \theta=0$ for all $t \in I$, where $\theta$ is a canonical contact 1 -form on the unit tangent bundle $T_{1} \mathbb{R}^{2}=\mathbb{R}^{2} \times S^{1}$ (cf. [1, 2]). This condition is equivalent to $\dot{\gamma}(t) \cdot \nu(t)=0$ for all $t \in I$. We say that $\gamma: I \rightarrow \mathbb{R}^{2}$ is a frontal if there exists a smooth mapping $\nu: I \rightarrow S^{1}$ such that $(\gamma, \nu)$ is a Legendre curve.

Let $(\gamma, \nu): I \rightarrow \mathbb{R}^{2} \times S^{1}$ be a Legendre curve. Then we have the Frenet formula of the frontal $\gamma$ as follows. We put $\boldsymbol{\mu}(t)=J(\nu(t))$, where $J$ is the anti-clockwise rotation by $\pi / 2$ on $\mathbb{R}^{2}$. We call the pair $\{\nu(t), \boldsymbol{\mu}(t)\}$ a moving frame along the frontal $\gamma(t)$ in $\mathbb{R}^{2}$ and the Frenet formula of the frontal (or, the Legendre curve) which is given by

$$
\left(\begin{array}{c}
\dot{\nu}(t) \\
\dot{\boldsymbol{\mu}}(t)
\end{array}\right)=\left(\begin{array}{cc}
0 & \ell(t) \\
-\ell(t) & 0
\end{array}\right)\left(\begin{array}{c}
\nu(t) \\
\boldsymbol{\mu}(t)
\end{array}\right)
$$

where $\ell(t)=\dot{\nu}(t) \cdot \boldsymbol{\mu}(t)$. Moreover, there exists a smooth function $\beta(t)$ such that

$$
\dot{\gamma}(t)=\beta(t) \boldsymbol{\mu}(t)
$$

We say that the pair of functions $(\ell, \beta)$ is the curvature of the Legendre curve $(\gamma, \nu): I \rightarrow$ $\mathbb{R}^{2} \times S^{1}$.

Now we consider the central and the canonical projections to give relationships between Legendre curves in $T_{1} S^{2}$ and $T_{1} \mathbb{R}^{2}$. We denote a hemisphere $S^{+}=\left\{(x, y, z) \in S^{2} \mid z>0\right\}$. 
We consider the central projection $\Phi: S^{+} \rightarrow \mathbb{R}^{2}$ by

$$
\Phi(x, y, z)=\left(\frac{x}{z}, \frac{y}{z}\right) .
$$

The central projection is useful to analyze the pedal curves (cf. [14, 15]).

Proposition 3.1 Let $(\gamma, \nu): I \rightarrow \Delta \subset S^{2} \times S^{2}$ be a spherical Legendre curve with the curvature $(m, n)$ and $\gamma(I) \subset S^{+}$. We denote $\gamma(t)=(x(t), y(t), z(t))$ and $\nu(t)=(a(t), b(t), c(t))$. Then $\widetilde{\gamma}=\Phi \circ \gamma$ is a frontal in $\mathbb{R}^{2}$. More preciously, $(\widetilde{\gamma}, \widetilde{\nu}): I \rightarrow \mathbb{R}^{2} \times S^{1}$ is a Legendre curve, where

$$
\widetilde{\gamma}(t)=\left(\frac{x(t)}{z(t)}, \frac{y(t)}{z(t)}\right), \widetilde{\nu}(t)=\frac{1}{\sqrt{a^{2}(t)+b^{2}(t)}}(a(t), b(t))
$$

with the curvature

$$
\ell(t)=\frac{n(t) z(t)}{a^{2}(t)+b^{2}(t)}, \beta(t)=\frac{m(t) z^{2}(t)+(x(t) b(t)-y(t) a(t)) \dot{z}(t)}{z^{2}(t) \sqrt{a^{2}(t)+b^{2}(t)}} .
$$

Proof. Since $(\gamma, \nu)$ is a spherical Legendre curve, we have

$$
x(t) a(t)+y(t) b(t)+z(t) c(t)=0, \dot{x}(t) a(t)+\dot{y}(t) b(t)+\dot{z}(t) c(t)=0 .
$$

It follows that $x(t) \dot{a}(t)+y(t) \dot{b}(t)+z(t) \dot{c}(t)=0$. By definition, we have

$$
\boldsymbol{\mu}(t)=\gamma(t) \times \nu(t)=(y(t) c(t)-z(t) b(t), z(t) a(t)-x(t) c(t), x(t) b(t)-y(t) a(t)) .
$$

By a direct calculation, we have

$$
\begin{aligned}
& m(t)=\dot{\gamma}(t) \cdot \boldsymbol{\mu}(t)=\frac{-\dot{x}(t) b(t)+\dot{y}(t) a(t)}{z(t)}, \\
& n(t)=\dot{\nu}(t) \cdot \boldsymbol{\mu}(t)=\frac{-\dot{a}(t) b(t)+a(t) \dot{b}(t)}{z(t)} .
\end{aligned}
$$

By the assumption $\gamma(t) \in S^{+}$, we have $c(t) \neq \pm 1$ and hence $a^{2}(t)+b^{2}(t) \neq 0$. It follows that $\widetilde{\nu}: I \rightarrow S^{1}, \widetilde{\nu}(t)=(a(t), b(t)) / \sqrt{a^{2}(t)+b^{2}(t)}$ is a smooth mapping. Moreover, we have

$$
\dot{\widetilde{\gamma}}(t)=\left(\frac{\dot{x}(t) z(t)-x(t) \dot{z}(t)}{z^{2}(t)}, \frac{\dot{y}(t) z(t)-y(t) \dot{z}(t)}{z^{2}(t)}\right)
$$

and $\dot{\vec{\gamma}}(t) \cdot \widetilde{\nu}(t)=0$. Therefore $(\widetilde{\gamma}, \widetilde{\nu}): I \rightarrow \mathbb{R}^{2} \times S^{1}$ is a Legendre curve.

By definition, we have $\widetilde{\boldsymbol{\mu}}(t)=J(\widetilde{\nu}(t))=(-b(t), a(t)) / \sqrt{a^{2}(t)+b^{2}(t)}$ and the curvature

$$
\begin{aligned}
\ell(t) & =\dot{\widetilde{\nu}}(t) \cdot \widetilde{\boldsymbol{\mu}}(t)=\frac{a(t) \dot{b}(t)-\dot{a}(t) b(t)}{a^{2}(t)+b^{2}(t)}=\frac{n(t) z(t)}{a^{2}(t)+b^{2}(t)}, \\
\beta(t) & =\dot{\tilde{\gamma}}(t) \cdot \widetilde{\boldsymbol{\mu}}(t)=\frac{(-\dot{x}(t) b(t)+\dot{y}(t) a(t)) z(t)+(x(t) b(t)-y(t) a(t)) \dot{z}(t)}{z^{2}(t) \sqrt{a^{2}(t)+b^{2}(t)}} \\
& =\frac{m(t) z^{2}(t)+(x(t) b(t)-y(t) a(t)) \dot{z}(t)}{z^{2}(t) \sqrt{a^{2}(t)+b^{2}(t)}} .
\end{aligned}
$$

Also, we consider the canonical projection $\pi: S^{+} \rightarrow D^{2} \subset \mathbb{R}^{2}$ by $\pi(x, y, z)=(x, y)$, where $D^{2}=\left\{(x, y) \in \mathbb{R}^{2} \mid x^{2}+y^{2}<1\right\}$. 
Proposition 3.2 Let $(\gamma, \nu): I \rightarrow \Delta \subset S^{2} \times S^{2}$ be a spherical Legendre curve with the curvature $(m, n)$ and $\gamma(I) \subset S^{+}$. We denote $\gamma(t)=(x(t), y(t), z(t))$ and $\nu(t)=(a(t), b(t), c(t))$. Then $\widetilde{\gamma}=\pi \circ \gamma$ is a frontal in $D^{2} \subset \mathbb{R}^{2}$. More preciously, $(\widetilde{\gamma}, \widetilde{\nu}): I \rightarrow D^{2} \times S^{1}$ is a Legendre curve, where

$$
\widetilde{\gamma}(t)=(x(t), y(t)), \widetilde{\nu}(t)=\frac{(z(t) a(t)-x(t) c(t), z(t) b(t)-y(t) c(t))}{\sqrt{(z(t) a(t)-x(t) c(t))^{2}+(z(t) b(t)-y(t) c(t))^{2}}}
$$

with the curvature

$$
\begin{aligned}
\ell(t) & =\frac{n(t) z(t)+x(t) \dot{y}(t)-\dot{x}(t) y(t)}{(z(t) a(t)-x(t) c(t))^{2}+(z(t) b(t)-y(t) c(t))^{2}} \\
\beta(t) & =\frac{m(t)-(x(t) b(t)-y(t) a(t)) \dot{z}(t)}{\sqrt{(z(t) a(t)-x(t) c(t))^{2}+(z(t) b(t)-y(t) c(t))^{2}}} .
\end{aligned}
$$

Proof. If $z(t) a(t)-x(t) c(t)=0$ and $z(t) b(t)-y(t) c(t)=0$, then $a(t)=x(t) c(t) / z(t)$ and $b(t)=y(t) c(t) / z(t)$. Since $\nu(t) \in S^{2}$, we have $c^{2}(t)=z^{2}(t)$ and hence $c(t)= \pm z(t)$. It follows that $a(t)= \pm x(t)$ and $b(t)= \pm y(t)$. It is contradict the fact that $\gamma(t) \cdot \nu(t)=0$. Hence $\widetilde{\nu}$ is a smooth mapping. By $\dot{x}(t) a(t)+\dot{y}(t) b(t)+\dot{z}(t) c(t)=0$ and $\dot{x}(t) x(t)+\dot{y}(t) y(t)+\dot{z}(t) z(t)=0$, we have $\dot{\widetilde{\gamma}}(t) \cdot \widetilde{\nu}(t)=0$. Therefore $(\widetilde{\gamma}, \widetilde{\nu}): I \rightarrow D^{2} \times S^{1}$ is a Legendre curve. By a similar calculation as in Proposition 3.1, we have the curvature $(\ell, \beta)$ of the Legendre curve $(\widetilde{\gamma}, \widetilde{\nu})$.

Remark 3.3 As a projection from the sphere to the plane, how about the stereographic projection. The properties of the stereographic projection see $[11,18,19]$, for example. Does it hold the similar results of Propositions 3.1 and 3.2 or not?

Conversely, for a Legendre curve in the unit tangent bundle, we have a spherical Legendre curve as follows.

Proposition 3.4 Let $(\widetilde{\gamma}, \widetilde{\nu}): I \rightarrow \mathbb{R}^{2} \times S^{1}$ be a Legendre curve with the curvature $(\ell, \beta)$. We denote $\widetilde{\gamma}(t)=(x(t), y(t))$ and $\widetilde{\nu}(t)=(a(t), b(t))$. Then $\gamma=\Phi^{-1} \circ \widetilde{\gamma}$ is a frontal in $S^{+}$. More preciously, $(\gamma, \nu): I \rightarrow \Delta \subset S^{+} \times S^{2}$ is a spherical Legendre curve, where

$$
\gamma(t)=\frac{(x(t), y(t), 1)}{\sqrt{1+x^{2}(t)+y^{2}(t)}}, \nu(t)=\frac{(a(t), b(t),-(x(t) a(t)+y(t) b(t)))}{\sqrt{1+(x(t) a(t)+y(t) b(t))^{2}}}
$$

with the curvature

$$
m(t)=\frac{\beta(t)+(x(t) \dot{y}(t)-\dot{x}(t) y(t))(x(t) a(t)+y(t) b(t))}{\left(1+x^{2}(t)+y^{2}(t)\right) \sqrt{1+(x(t) a(t)+y(t) b(t))^{2}}}, n(t)=\frac{\ell(t) \sqrt{1+x^{2}(t)+y^{2}(t)}}{1+(x(t) a(t)+y(t) b(t))^{2}} .
$$

Proof. Since $(\widetilde{\gamma}, \widetilde{\nu}): I \rightarrow \mathbb{R}^{2} \times S^{1}$ is a Legendre curve, we have $\dot{x}(t) a(t)+\dot{y}(t) b(t)=0$. By definition, we have $\widetilde{\boldsymbol{\mu}}(t)=J(\widetilde{\nu}(t))=(-b(t), a(t))$. It follows that

$$
\ell(t)=-\dot{a}(t) b(t)+a(t) \dot{b}(t), \beta(t)=-\dot{x}(t) b(t)+\dot{y}(t) a(t) .
$$

By a direct calculation, we have

$$
\begin{aligned}
\dot{\gamma}(t)= & \frac{1}{1+x^{2}(t)+y^{2}(t)}\left(\left(1+y^{2}(t)\right) \dot{x}(t)-x(t) y(t) \dot{y}(t),\right. \\
& \left.\left.\left(1+x^{2}(t)\right) \dot{y}(t)-x(t) \dot{x}(t) y(t)\right),-(x(t) \dot{x}(t)+y(t) \dot{y}(t))\right) .
\end{aligned}
$$


Then $\gamma(t) \cdot \nu(t)=0$ and $\dot{\gamma}(t) \cdot \nu(t)=0$ for all $t \in I$. Therefore $(\gamma, \nu): I \rightarrow \Delta \subset S^{+} \times S^{2}$ is a spherical Legendre curve. By definition, we have

$$
\begin{aligned}
\boldsymbol{\mu}(t) & =\gamma(t) \times \nu(t) \\
& =\frac{\left(-x(t) y(t) a(t)-\left(1+y^{2}(t)\right) b(t),\left(1+x^{2}(t)\right) a(t)+x(t) y(t) b(t), x(t) b(t)-y(t) a(t)\right)}{\sqrt{\left(1+x^{2}(t)+y^{2}(t)\right)\left(1+(x(t) a(t)+y(t) b(t))^{2}\right)}}
\end{aligned}
$$

and the curvature

$$
\begin{aligned}
m(t) & =\dot{\gamma}(t) \cdot \boldsymbol{\mu}(t)=\frac{(x(t) \dot{y}(t)-\dot{x}(t) y(t))(x(t) a(t)+y(t) b(t))-\dot{x}(t) b(t)+\dot{y}(t) a(t)}{\left(1+x^{2}(t)+y^{2}(t)\right) \sqrt{1+(x(t) a(t)+y(t) b(t))^{2}}} \\
& =\frac{\beta(t)+(x(t) \dot{y}(t)-\dot{x}(t) y(t))(x(t) a(t)+y(t) b(t))}{\left(1+x^{2}(t)+y^{2}(t)\right) \sqrt{1+(x(t) a(t)+y(t) b(t))^{2}}}, \\
n(t) & =\dot{\nu}(t) \cdot \boldsymbol{\mu}(t)=\frac{(a(t) \dot{b}(t)-\dot{a}(t) b(t)) \sqrt{1+x^{2}(t)+y^{2}(t)}}{1+(x(t) a(t)+y(t) b(t))^{2}} \\
& =\frac{\ell(t) \sqrt{1+x^{2}(t)+y^{2}(t)}}{1+(x(t) a(t)+y(t) b(t))^{2}} .
\end{aligned}
$$

Proposition 3.5 Let $(\widetilde{\gamma}, \widetilde{\nu}): I \rightarrow D^{2} \times S^{1}$ be a Legendre curve with the curvature $(\ell, \beta)$. We denote $\widetilde{\gamma}(t)=(x(t), y(t))$ and $\widetilde{\nu}(t)=(a(t), b(t))$. Then $\gamma=\pi^{-1} \circ \widetilde{\gamma}$ is a frontal in $S^{+}$. More preciously, $(\gamma, \nu): I \rightarrow \Delta \subset S^{+} \times S^{2}$ is a spherical Legendre curve, where

$$
\begin{aligned}
\gamma(t)= & (x(t), y(t), z(t)), \\
\nu(t)= & \frac{1}{\sqrt{1-(x(t) a(t)+y(t) b(t))^{2}}}(a(t)-x(t)(x(t) a(t)-y(t) b(t)), \\
& b(t)-y(t)(x(t) a(t)-y(t) b(t)),-z(t)(x(t) a(t)+y(t) b(t)))
\end{aligned}
$$

with the curvature

$$
\begin{aligned}
m(t) & =\frac{\beta(t)+(\dot{x}(t) y(t)-x(t) \dot{y}(t))(x(t) a(t)-y(t) b(t))}{z(t) \sqrt{1-(x(t) a(t)+y(t) b(t))^{2}}}, \\
n(t) & =\frac{\ell(t) z^{2}(t)-\beta(t)(x(t) a(t)+y(t) b(t))+(x(t) \dot{y}(t)-\dot{x}(t) y(t))(x(t) a(t)+y(t) b(t))^{2}}{z(t)\left(1-(x(t) a(t)+y(t) b(t))^{2}\right)} .
\end{aligned}
$$

Here we put $z(t)=\sqrt{1-x^{2}(t)-y^{2}(t)}$.

Proof. Since $\widetilde{\gamma}(t) \cdot \widetilde{\gamma}(t)<1$ and $\widetilde{\nu}(t) \cdot \widetilde{\nu}(t)=1$, we have $x(t) a(t)+y(t) b(t)<1$ for all $t \in I$. Therefore $\nu: I \rightarrow S^{2}$ is a smooth mapping. By the same argument as in Proposition 3.4, we have

$$
\ell(t)=-\dot{a}(t) b(t)+a(t) \dot{b}(t), \beta(t)=-\dot{x}(t) b(t)+\dot{y}(t) a(t) .
$$

Since

$$
\dot{\gamma}(t)=\left(\dot{x}(t), \dot{y}(t),-\frac{x(t) \dot{x}(t)-y(t) \dot{y}(t)}{z(t)}\right)
$$


and $x^{2}(t)+y^{2}(t)+z^{2}(t)=1$, we have $\gamma(t) \cdot \nu(t)=0$ and $\dot{\gamma}(t) \cdot \nu(t)=0$ for all $t \in I$. Therefore $(\gamma, \nu): I \rightarrow \Delta \subset S^{+} \times S^{2}$ is a spherical Legendre curve. By definition, we have

$$
\boldsymbol{\mu}(t)=\frac{1}{\sqrt{1-(x(t) a(t)+y(t) b(t))^{2}}}(-z(t) b(t), z(t) a(t), x(t) b(t)-y(t) a(t))
$$

and the curvature $(m, n)$ of the spherical Legendre curve $(\gamma, \nu)$.

Second, we discuss relationships between framed curves in the Euclidean space and spherical Legendre curves.

We review on the framed curves in the unit tangent bundle, for more detail see [12]. We say that $\left(\gamma, \nu_{1}, \nu_{2}\right): I \rightarrow \mathbb{R}^{3} \times S^{2} \times S^{2}$ is a framed curve if

$$
\dot{\gamma}(t) \cdot \nu_{1}(t)=0, \dot{\gamma}(t) \cdot \nu_{2}(t)=0, \nu_{1}(t) \cdot \nu_{2}(t)=0
$$

for all $t \in I$. Then $\left(\nu_{1}, \nu_{2}\right) \in \Delta$.

Let $\left(\gamma, \nu_{1}, \nu_{2}\right): I \rightarrow \mathbb{R}^{3} \times \Delta$ be a framed curve and denote $\boldsymbol{\mu}(t)=\nu_{1}(t) \times \nu_{2}(t)$. The Frenet Serret type formula is given by

$$
\left(\begin{array}{c}
\dot{\nu_{1}}(t) \\
\dot{\nu_{2}}(t) \\
\dot{\boldsymbol{\mu}}(t)
\end{array}\right)=\left(\begin{array}{ccc}
0 & \ell(t) & m(t) \\
-\ell(t) & 0 & n(t) \\
-m(t) & -n(t) & 0
\end{array}\right)\left(\begin{array}{c}
\nu_{1}(t) \\
\nu_{2}(t) \\
\boldsymbol{\mu}(t)
\end{array}\right),
$$

where $\ell(t)=\dot{\nu_{1}}(t) \cdot \nu_{2}(t), m(t)=\dot{\nu_{1}}(t) \cdot \boldsymbol{\mu}(t)$ and $n(t)=\dot{\nu_{2}}(t) \cdot \boldsymbol{\mu}(t)$. Moreover, there exists a smooth mapping $\alpha: I \rightarrow \mathbb{R}$ such that

$$
\dot{\gamma}(t)=\alpha(t) \boldsymbol{\mu}(t)
$$

We say that the pair of the functions $(\ell, m, n, \alpha)$ is the curvature of the framed curve $\left(\gamma, \nu_{1}, \nu_{2}\right): I \rightarrow \mathbb{R}^{3} \times \Delta$.

Let $\left(\gamma, \nu_{1}, \nu_{2}\right): I \rightarrow \mathbb{R}^{3} \times \Delta$ be a framed curve with the curvature of the framed curve $(\ell, m, n, \alpha)$. For the normal plane of $\gamma(t)$, spanned by $\nu_{1}(t)$ and $\nu_{2}(t)$, there is some ambient of framed curves similarly to the case of the Bishop frame of a regular space curve (cf. [4]). We define $\left(\bar{\nu}_{1}(t), \bar{\nu}_{2}(t)\right) \in \Delta$ by

$$
\left(\begin{array}{c}
\bar{\nu}_{1}(t) \\
\bar{\nu}_{2}(t)
\end{array}\right)=\left(\begin{array}{cc}
\cos \theta(t) & -\sin \theta(t) \\
\sin \theta(t) & \cos \theta(t)
\end{array}\right)\left(\begin{array}{l}
\nu_{1}(t) \\
\nu_{2}(t)
\end{array}\right)
$$

where $\theta(t)$ is a smooth function. Then $\left(\gamma, \bar{\nu}_{1}, \bar{\nu}_{2}\right): I \rightarrow \mathbb{R}^{3} \times \Delta$ is also a framed curve and

$$
\begin{aligned}
\overline{\boldsymbol{\mu}}(t) & =\bar{\nu}_{1}(t) \times \bar{\nu}_{2}(t) \\
& =\left(\cos \theta(t) \nu_{1}(t)-\sin \theta(t) \nu_{2}(t)\right) \times\left(\sin \theta(t) \nu_{1}(t)+\cos \theta(t) \nu_{2}(t)\right) \\
& =\nu_{1}(t) \times \nu_{2}(t)=\boldsymbol{\mu}(t)
\end{aligned}
$$

By a direct calculation, we have

$$
\begin{aligned}
\dot{\bar{\nu}}_{1}(t)= & (\ell(t)-\dot{\theta}(t)) \sin \theta(t) \nu_{1}(t)+(\ell(t)-\dot{\theta}(t)) \cos \theta(t) \nu_{2}(t) \\
& +(m(t) \cos \theta(t)-n(t) \sin \theta(t)) \boldsymbol{\mu}(t), \\
\dot{\bar{\nu}}_{2}(t)= & -(\ell(t)-\dot{\theta}(t)) \cos \theta(t) \nu_{1}(t)+(\ell(t)-\dot{\theta}(t)) \sin \theta(t) \nu_{2}(t) \\
& +(m(t) \sin \theta(t)+n(t) \cos \theta(t)) \boldsymbol{\mu}(t) .
\end{aligned}
$$


If we take a smooth function $\theta: I \rightarrow \mathbb{R}$ which satisfies $\dot{\theta}(t)=\ell(t)$, then we call the frame $\left\{\bar{\nu}_{1}(t), \bar{\nu}_{2}(t), \boldsymbol{\mu}(t)\right\}$ an adapted frame along the framed base curve $\gamma(t)$. It follows that the Frenet Serret type formula is given by

$$
\left(\begin{array}{c}
\dot{\bar{\nu}}_{1}(t) \\
\dot{\bar{\nu}}_{2}(t) \\
\dot{\boldsymbol{\mu}}(t)
\end{array}\right)=\left(\begin{array}{ccc}
0 & 0 & \bar{m}(t) \\
0 & 0 & \bar{n}(t) \\
-\bar{m}(t) & -\bar{n}(t) & 0
\end{array}\right)\left(\begin{array}{c}
\bar{\nu}_{1}(t) \\
\bar{\nu}_{2}(t) \\
\boldsymbol{\mu}(t)
\end{array}\right)
$$

where $\bar{m}(t)$ and $\bar{n}(t)$ are given by

$$
\left(\begin{array}{c}
\bar{m}(t) \\
\bar{n}(t)
\end{array}\right)=\left(\begin{array}{cc}
\cos \theta(t) & -\sin \theta(t) \\
\sin \theta(t) & \cos \theta(t)
\end{array}\right)\left(\begin{array}{c}
m(t) \\
n(t)
\end{array}\right) .
$$

Proposition 3.6 Let $\left(\gamma, \nu_{1}, \nu_{2}\right): I \rightarrow \mathbb{R}^{3} \times \Delta$ be a framed curve with the curvature $(\ell, m, n, \alpha)$.

(1) Suppose that $\left\{\bar{\nu}_{1}(t), \bar{\nu}_{2}(t), \boldsymbol{\mu}(t)\right\}$ is an adapted frame of $\gamma(t)$. Then $\left(\bar{\nu}_{1}, \bar{\nu}_{2}\right): I \rightarrow \Delta \subset$ $S^{2} \times S^{2}$ is a spherical Legendre curve with the curvature $(\bar{m}(t), \bar{n}(t))$.

(2) Let $\gamma(t)$ be non-zero. We denote $\widetilde{\gamma}(t)=\gamma(t) /|\gamma(t)|$ and $\widetilde{\gamma}(t)=a(t) \nu_{1}(t)+b(t) \nu_{2}(t)+$ $c(t) \boldsymbol{\mu}(t)$ with $a^{2}(t)+b^{2}(t)+c^{2}(t)=1$. Suppose that $a^{2}(t)+b^{2}(t) \neq 0$. Then $\widetilde{\gamma}(t)$ is a frontal in $S^{2}$. More preciously, $(\widetilde{\gamma}, \widetilde{\nu}): I \rightarrow \Delta \subset S^{2} \times S^{2}$ is a spherical Legendre curve, where

$$
\widetilde{\nu}(t)=\frac{\widetilde{\gamma}(t) \times \boldsymbol{\mu}(t)}{|\widetilde{\gamma}(t) \times \boldsymbol{\mu}(t)|}
$$

with the curvature

$$
\begin{aligned}
\widetilde{m}(t) & =-\frac{a(t) m(t)+b(t) n(t)+\dot{c}(t)}{\sqrt{a^{2}(t)+b^{2}(t)}} \\
\widetilde{n}(t) & =\frac{\left(a^{2}(t)+b^{2}(t)\right)(a(t) n(t)-b(t) m(t)+c(t) \ell(t))+(a(t) \dot{b}(t)-\dot{a}(t) b(t)) c(t)}{\sqrt{a^{2}(t)+b^{2}(t)}} .
\end{aligned}
$$

Proof. (1) By definition, $\left(\bar{\nu}_{1}, \bar{\nu}_{2}\right)$ is a spherical Legendre curve with the curvature $(\bar{m}(t), \bar{n}(t))$.

(2) Since $\widetilde{\nu}(t)=\left(b(t) \nu_{1}(t)-a(t) \nu_{2}(t)\right) / \sqrt{a^{2}(t)+b^{2}(t)}$, we have

$$
\widetilde{\boldsymbol{\mu}}(t)=\frac{1}{\sqrt{a^{2}(t)+b^{2}(t)}}\left(a(t) c(t) \nu_{1}(t)+b(t) c(t) \nu_{2}(t)-\left(a^{2}(t)+b^{2}(t)\right) \boldsymbol{\mu}(t)\right) .
$$

By using the Frenet Serret type formula, we have

$$
\begin{aligned}
\dot{\widetilde{\gamma}}(t)= & (\dot{a}(t)-b(t) \ell(t)-c(t) m(t)) \nu_{1}(t)+(\dot{b}(t)+a(t) \ell(t)-c(t) n(t)) \nu_{2}(t) \\
& +(\dot{c}(t)+a(t) m(t)+b(t) n(t)) \boldsymbol{\mu}(t), \\
\dot{\widetilde{\nu}}(t)= & \frac{1}{\left(a^{2}(t)+b^{2}(t)\right)^{\frac{3}{2}}}\left(\left(\dot{b}(t) a^{2}(t)+a(t)\left(a^{2}(t)+b^{2}(t)\right) \ell(t)-\dot{a}(t) a(t) b(t)\right) \nu_{1}(t)\right. \\
& +\left(-\dot{a}(t) b^{2}(t)+b(t)\left(a^{2}(t)+b^{2}(t)\right) \ell(t)+\dot{b}(t) a(t) b(t)\right) \nu_{2}(t) \\
& \left.+\left(a^{2}(t)+b^{2}(t)\right)(-a(t) n(t)+b(t) m(t)) \boldsymbol{\mu}(t)\right) .
\end{aligned}
$$

By a direct calculation, we have

$$
\begin{aligned}
\widetilde{m}(t) & =\dot{\widetilde{\gamma}}(t) \cdot \widetilde{\boldsymbol{\mu}}(t)=-\frac{a(t) m(t)+b(t) n(t)+\dot{c}(t)}{\sqrt{a^{2}(t)+b^{2}(t)}}, \\
\widetilde{n}(t) & =\dot{\widetilde{\nu}}(t) \cdot \widetilde{\boldsymbol{\mu}}(t) \\
& =\frac{\left(a^{2}(t)+b^{2}(t)\right)(a(t) n(t)-b(t) m(t)+c(t) \ell(t))+(a(t) \dot{b}(t)-\dot{a}(t) b(t)) c(t)}{\sqrt{a^{2}(t)+b^{2}(t)}} .
\end{aligned}
$$


Conversely, for a spherical Legendre curve, we have a framed curve as follows.

Proposition 3.7 Let $(\gamma, \nu): I \rightarrow \Delta \subset S^{2} \times S^{2}$ be a spherical Legendre curve with the curvature $(m, n)$. Then $(\gamma, \gamma, \nu): I \rightarrow S^{2} \times \Delta \subset \mathbb{R}^{3} \times \Delta$ is a framed curve with the curvature $(\ell, m, n, \alpha)=$ $(0, m, n, m)$.

Proof. Since $(\gamma, \nu): I \rightarrow \Delta \subset S^{2} \times S^{2}$ is a spherical Legendre curve, $(\gamma, \gamma, \nu): I \rightarrow S^{2} \times \Delta \subset$ $\mathbb{R}^{3} \times \Delta$ is a framed curve with the curvature $(0, m, n, m)$.

\section{Evolutes of fronts in the sphere}

In this section, we assume that $(\gamma, \nu): I \rightarrow \Delta \subset S^{2} \times S^{2}$ is a Legendre immersion. It follows that $(m(t), n(t)) \neq(0,0)$ for all $t \in I$. We define an evolute of the front and give properties of the evolute in the sphere. For the evolutes of curves in the Euclidean plane see $[5,7,8,10]$.

Definition 4.1 We define an evolute $\mathcal{E} v(\gamma): I \rightarrow S^{2}$ of the front $\gamma$ by

$$
\mathcal{E} v(\gamma)(t)= \pm \frac{n(t)}{\sqrt{m^{2}(t)+n^{2}(t)}} \gamma(t) \mp \frac{m(t)}{\sqrt{m^{2}(t)+n^{2}(t)}} \nu(t)
$$

Remark 4.2 If $(\gamma, \nu)$ is a Legendre immersion with the curvature $(m, n)$, then $(\gamma,-\nu)$ (respectively, $(-\gamma, \nu))$ is a Legendre immersion with the curvature $(-m, n)$ (respectively, $(m,-n))$ by Remark 2.3. It is easy to see that the evolute $\mathcal{E} v(\gamma)$ does not change. For the case $(\nu, \gamma)$, see below Corollary 4.6.

Proposition 4.3 Let $\gamma: I \rightarrow S^{2}$ be a regular curve. Then the evolute of the regular curve and the evolute of the front are coincide.

Proof. We consider a Legendre immersion $(\gamma, \boldsymbol{n}): I \rightarrow \Delta \subset S^{2} \times S^{2}$ with the curvature $(m, n)$, see Example 2.7. Since $\boldsymbol{n}(t)=\nu(t)$ and $\boldsymbol{t}(t)=-\boldsymbol{\mu}(t)$, we have $m(t)<0$. The geodesic curvature of the regular curve is given by $\kappa_{g}(t)=n(t) /|m(t)|=-n(t) / m(t)$. By the definition of the evolute of the regular curve (1), we have

$$
\begin{aligned}
E v(\gamma)(t) & = \pm \frac{\kappa_{g}(t)}{\sqrt{\kappa_{g}^{2}(t)+1}} \gamma(t) \pm \frac{1}{\sqrt{\kappa_{g}^{2}(t)+1}} \boldsymbol{n}(t) \\
& = \pm \frac{n(t)}{\sqrt{m^{2}(t)+n^{2}(t)}} \gamma(t) \mp \frac{m(t)}{\sqrt{m^{2}(t)+n^{2}(t)}} \nu(t)=\mathcal{E} v(\gamma)(t)
\end{aligned}
$$

Proposition 4.4 Suppose that $(\gamma, \nu): I \rightarrow \Delta \subset S^{2} \times S^{2}$ and $(\widetilde{\gamma}, \widetilde{\nu}): \widetilde{I} \rightarrow \Delta \subset S^{2} \times S^{2}$ are parametrically equivalent via the change of parameter $t: \widetilde{I} \rightarrow I$. Then $\mathcal{E} v(\widetilde{\gamma})(u)=\mathcal{E} v(\gamma)(t(u))$.

Proof. We denote $(m(t), n(t))$ and $(\widetilde{m}(u), \widetilde{n}(u))$ the curvature of $(\gamma(t), \nu(t))$ and $(\widetilde{\gamma}(u), \widetilde{\nu}(u))$ respectively. By the assumption, we have $(\widetilde{\gamma}(u), \widetilde{\nu}(u))=(\gamma(t(u)), \nu(t(u)))$. By using the relationship between the curvature $(2)$, we have $\mathcal{E} v(\widetilde{\gamma})(u)=\mathcal{E} v(\gamma)(t(u))$. 
Proposition 4.5 Let $\theta \in[0,2 \pi)$ and $\left(\gamma_{\theta}, \nu_{\theta}\right): I \rightarrow \Delta \subset S^{2} \times S^{2}$ be a parallel Legendre immersion of $(\gamma, \nu)$. Then the evolute of the parallel curve and the evolute of the front are coincide.

Proof. By Proposition 2.9, $\left(m_{\theta}(t), n_{\theta}(t)\right)=(m(t) \cos \theta+n(t) \sin \theta,-m(t) \cos \theta+n(t) \cos \theta)$ is the curvature of $\left(\gamma_{\theta}, \nu_{\theta}\right)$. Then we have $m_{\theta}^{2}(t)+n_{\theta}^{2}(t)=m^{2}(t)+n^{2}(t)$. It follows that

$$
\begin{aligned}
\mathcal{E} v\left(\gamma_{\theta}\right)(t)= & \pm \frac{n_{\theta}(t)}{\sqrt{m_{\theta}^{2}(t)+n_{\theta}^{2}(t)}} \gamma_{\theta}(t) \mp \frac{m_{\theta}(t)}{\sqrt{m_{\theta}^{2}(t)+n_{\theta}^{2}(t)}} \nu_{\theta}(t) \\
= & \pm \frac{(-m(t) \sin \theta+n(t) \cos \theta)}{\sqrt{m^{2}(t)+n^{2}(t)}}(\cos \theta \gamma(t)+\sin \theta \nu(t)) \\
& \mp \frac{(m(t) \cos \theta+n(t) \sin \theta)}{\sqrt{m^{2}(t)+n^{2}(t)}}(-\sin \theta \gamma(t)+\cos \theta \nu(t)) \\
= & \pm \frac{n(t)}{\sqrt{m^{2}(t)+n^{2}(t)}} \gamma(t) \mp \frac{m(t)}{\sqrt{m^{2}(t)+n^{2}(t)}} \nu(t)=\mathcal{E} v(\gamma)(t) .
\end{aligned}
$$

If we take $\theta=\pi / 2$, then $\left(\gamma_{\theta}, \nu_{\theta}\right)=(\nu,-\gamma)$. By Proposition 4.5 and Remark 4.2 , we have the following Corollary.

Corollary 4.6 For a Legendre immersion $(\gamma, \nu): I \rightarrow \Delta \subset S^{2} \times S^{2},(\nu, \gamma)$ is also a Legendre immersion. Then the evolute of the front and the evolute of the dual curve are coincide, that $i s, \mathcal{E} v(\gamma)(t)=\mathcal{E} v(\nu)(t)$.

We define a family of functions $H: I \times S^{2} \rightarrow \mathbb{R}$ by

$$
H(t, \boldsymbol{v})=\boldsymbol{\mu}(t) \cdot \boldsymbol{v}
$$

Proposition 4.7 Let $(\gamma, \nu): I \rightarrow \Delta \subset S^{2} \times S^{2}$ be a Legendre immersion with the curvature $(m, n)$. We have the following.

(1) $H(t, \boldsymbol{v})=0$ if and only if $\boldsymbol{v}=a \gamma(t)+b \nu(t)$ for some $a, b \in \mathbb{R}$ with $a^{2}+b^{2}=1$.

(2) $H(t, \boldsymbol{v})=\frac{\partial H}{\partial t}(t, \boldsymbol{v})=0$ if and only if $\boldsymbol{v}= \pm \frac{n(t)}{\sqrt{m^{2}(t)+n^{2}(t)}} \gamma(t) \mp \frac{m(t)}{\sqrt{m^{2}(t)+n^{2}(t)}} \nu(t)$.

Proof. (1) Since $\{\gamma(t), \nu(t), \boldsymbol{\mu}(t)\}$ is an orthogonal base on $\mathbb{R}^{3}$, we have $\boldsymbol{\mu}(t) \cdot \boldsymbol{v}=0$ if and only if there exist real numbers $a, b \in \mathbb{R}$ such that $\boldsymbol{v}=a \gamma(t)+b \nu(t) \in S^{2}$.

(2) Since $(d / d t) H(t, \boldsymbol{v})=(-m(t) \gamma(t)-n(t) \nu(t)) \cdot \boldsymbol{v}$, we have $a^{2}+b^{2}=1$ and $a m(t)+b n(t)=$ 0 . It follows that

$$
a= \pm \frac{n(t)}{\sqrt{m^{2}(t)+n^{2}(t)}}, b=\mp \frac{m(t)}{\sqrt{m^{2}(t)+n^{2}(t)}} .
$$

By a direct calculation, the converse holds.

We can show that $H$ is a Morse family, in the sense of Legendrian singularity theory (cf. $[1,3,13,22])$, namely, $(H, \partial H / \partial t): I \times S^{2} \rightarrow \mathbb{R} \times \mathbb{R}$ is a submersion at $(t, \boldsymbol{v}) \in D(H)$, where

$$
D(H)=\{(t, \boldsymbol{v}) \mid H(t, \boldsymbol{v})=(\partial H / \partial t)(t, \boldsymbol{v})=0\} .
$$

It follows that the evolute of the front $\mathcal{E} v(\gamma)$ is a (wave) front of a Legendre immersion and is given by the envelope of the parallel of the front. In fact, we have the following. 
Proposition 4.8 Let $(\gamma, \nu): I \rightarrow \Delta \subset S^{2} \times S^{2}$ be a Legendre immersion with the curvature $(m, n)$. Then $\mathcal{E} v(\gamma)$ is a front. More precisely, $(\mathcal{E} v(\gamma), \boldsymbol{\mu}): I \rightarrow \Delta \subset S^{2} \times S^{2}$ is a Legendre immersion with the curvature

$$
m_{\mathcal{E} v}(t)=\frac{\dot{m}(t) n(t)-m(t) \dot{n}(t)}{m^{2}(t)+n^{2}(t)}, n_{\mathcal{E} v}(t)= \pm \sqrt{m^{2}(t)+n^{2}(t)} .
$$

Proof. Here we denote $\left(\gamma_{\mathcal{E} v}, \nu_{\mathcal{E} v}\right)=(\mathcal{E} v(\gamma), \boldsymbol{\mu})$. By definition of the evolute of the front $\mathcal{E} v(t)$, $\gamma_{\mathcal{E} v}(t) \cdot \nu_{\mathcal{E} v}(t)=0$ for all $t \in I$. Moreover, since

$$
\dot{\gamma}_{\mathcal{E} v}(t)= \pm \frac{d}{d t}\left(\frac{n(t)}{\sqrt{m^{2}(t)+n^{2}(t)}}\right) \gamma(t) \mp \frac{d}{d t}\left(\frac{m(t)}{\sqrt{m^{2}(t)+n^{2}(t)}}\right) \nu(t),
$$

we have $\dot{\gamma}_{\mathcal{E} v}(t) \cdot \nu_{\mathcal{E} v}(t)=0$ for all $t \in I$. Hence $\left(\gamma_{\mathcal{E} v}, \nu_{\mathcal{E} v}\right): I \rightarrow \Delta \subset S^{2} \times S^{2}$ is a Legendre curve. By a direct calculation, we have $\dot{\nu}_{\mathcal{E} v}(t)=\dot{\boldsymbol{\mu}}(t)=-m(t) \gamma(t)-n(t) \nu(t)$ and

$$
\boldsymbol{\mu}_{\mathcal{E} v}(t)=\gamma_{\mathcal{E} v}(t) \times \nu_{\mathcal{E} v}(t)=\mp \frac{m(t)}{\sqrt{m^{2}(t)+n^{2}(t)}} \gamma(t) \mp \frac{n(t)}{\sqrt{m^{2}(t)+n^{2}(t)}} \nu(t) .
$$

Then the curvature is given by

$$
\begin{aligned}
m_{\mathcal{E} v}(t) & =\dot{\gamma}_{\mathcal{E} v}(t) \cdot \boldsymbol{\mu}_{\mathcal{E} v}(t) \\
& =-\frac{m(t)}{\sqrt{m^{2}(t)+n^{2}(t)}} \frac{d}{d t}\left(\frac{n(t)}{\sqrt{m^{2}(t)+n^{2}(t)}}\right)+\frac{n(t)}{\sqrt{m^{2}(t)+n^{2}(t)}} \frac{d}{d t}\left(\frac{m(t)}{\sqrt{m^{2}(t)+n^{2}(t)}}\right) \\
& =\frac{\dot{m}(t) n(t)-m(t) \dot{n}(t)}{m^{2}(t)+n^{2}(t)} \\
n_{\mathcal{E} v}(t) & =\dot{\nu}_{\mathcal{E} v}(t) \cdot \boldsymbol{\mu}_{\mathcal{E} v}(t) \\
& = \pm \frac{m^{2}(t)}{\sqrt{m^{2}(t)+n^{2}(t)}} \pm \frac{n^{2}(t)}{\sqrt{m^{2}(t)+n^{2}(t)}}= \pm \sqrt{m^{2}(t)+n^{2}(t)}
\end{aligned}
$$

It follows from $n_{\mathcal{E} v}(t) \neq 0$ for all $t \in I$ that $\left(\gamma_{\mathcal{E} v}, \nu_{\mathcal{E} v}\right)$ is a Legendre immersion.

Remark 4.9 The evolute of $\gamma$ and of $\nu$ are coincide by Corollary 4.6. It follows that the evolute of $\boldsymbol{\mu}$ is given by the second evolute of $\gamma$, see Theorem 4.11 below, that is, $\mathcal{E} v(\boldsymbol{\mu})=\mathcal{E} v(\mathcal{E} v(\gamma))$ by Proposition 4.8

We denote a plane by $P(\boldsymbol{v}, a)=\left\{\boldsymbol{x} \in \mathbb{R}^{3} \mid \boldsymbol{x} \cdot \boldsymbol{v}=a\right\}$, where $\boldsymbol{v} \in S^{2}$ is a constant vector and $a \in \mathbb{R}$ is a constant.

Proposition 4.10 Let $(\gamma, \nu): I \rightarrow \Delta \subset S^{2} \times S^{2}$ be a Legendre immersion with the curvature $(m, n)$. Then $\mathcal{E} v(\gamma)$ is constant if and only if there exist a vector $\boldsymbol{v} \in S^{2}$ and $a, b \in \mathbb{R}$ with $a^{2}+b^{2}=1$ such that $\gamma(t) \in P(\boldsymbol{v}, a) \cap S^{2}$ and $\nu(t) \in P(\boldsymbol{v}, b) \cap S^{2}$ for all $t \in I$.

Proof. By Proposition 4.8 and $\dot{\mathcal{E}} v(\gamma)(t)=0$, we have $\dot{m}(t) n(t)-m(t) \dot{n}(t)=0$ and $m^{2}(t)+$ $n^{2}(t) \neq 0$ for all $t \in I$. Then $m$ and $n$ are linearly dependent, that is, there exist $a, b \in \mathbb{R}$ with $a^{2}+b^{2}=1$ such that $a m(t)+b n(t)=0$ for all $t \in I$. By the Frenet Serret formula, we have $a \dot{\gamma}(t)+b \dot{\nu}(t)=0$ for all $t \in I$. There exists a constant vector $\boldsymbol{v} \in S^{2}$ such that $a \gamma(t)+b \nu(t)=\boldsymbol{v}$. Therefore, we have $\gamma(t) \in P(\boldsymbol{v}, a) \cap S^{2}$ and $\nu(t) \in P(\boldsymbol{v}, b) \cap S^{2}$ for all $t \in I$. 
Conversely, if $\gamma(t) \cdot \boldsymbol{v}=a$ and $\nu(t) \cdot \boldsymbol{v}=b$ for all $t \in I$, then $m(t) \boldsymbol{\mu}(t) \cdot \boldsymbol{v}=0$ and $n(t) \boldsymbol{\mu}(t) \cdot \boldsymbol{v}=0$. It follows that $\boldsymbol{\mu}(t) \cdot \boldsymbol{v}=0$. Since $\{\gamma(t), \nu(t), \boldsymbol{\mu}(t)\}$ is an orthogonal basis on $\mathbb{R}^{3}$, we can denote $\boldsymbol{v}=a \gamma(t)+b \nu(t)$. By differentiate, we have $a m(t)+b n(t)=0$ and $a \dot{m}(t)+b \dot{n}(t)=0$. Since $a^{2}+b^{2}=1$, we have $\dot{m}(t) n(t)-m(t) \dot{n}(t)=0$ for all $t \in I$. It follows that $\mathcal{E} v(\gamma)$ is constant.

Let $(\gamma, \nu): I \rightarrow \Delta \subset S^{2} \times S^{2}$ be a Legendre immersion with the curvature $(m, n)$. We give the form of the $k$-th evolute of the front, where $k$ is a natural number. We denote

$$
\mathcal{E} v^{0}(\gamma)(t)=\gamma(t), \nu_{0}(t)=\nu(t), \boldsymbol{\mu}_{0}(t)=\boldsymbol{\mu}(t), m_{0}=m(t), n_{0}=n(t),
$$

for convenience. We define

$$
\begin{aligned}
& \mathcal{E} v^{k}(\gamma)(t)=\mathcal{E} v\left(\mathcal{E} v^{k-1}(\gamma)\right)(t), \nu_{k}(t)=\boldsymbol{\mu}_{k-1}(t), \boldsymbol{\mu}_{k}(t)=\mathcal{E} v^{k}(\gamma)(t) \times \nu_{k}(t), \\
& m_{k}(t)=\frac{\dot{m}_{k-1}(t) n_{k-1}(t)-m_{k-1}(t) \dot{n}_{k-1}(t)}{m_{k-1}^{2}(t)+n_{k-1}^{2}(t)}, n_{k}(t)= \pm \sqrt{m_{k-1}^{2}(t)+n_{k-1}^{2}(t)},
\end{aligned}
$$

inductively. Then we have the following theorem.

Theorem 4.11 Let $(\gamma, \nu): I \rightarrow \Delta \subset S^{2} \times S^{2}$ be a Legendre immersion with the curvature $(m, n)$. Then $\mathcal{E} v^{k}(\gamma)$ is a front. More precisely, $\left(\mathcal{E} v^{k}(\gamma), \nu_{k}\right): I \rightarrow \Delta \subset S^{2} \times S^{2}$ is a Legendre immersion with the curvature $\left(m_{k}, n_{k}\right)$, where

$$
\mathcal{E} v^{k}(\gamma)(t)= \pm \frac{n_{k-1}(t)}{\sqrt{m_{k-1}^{2}(t)+n_{k-1}^{2}(t)}} \mathcal{E} v^{k-1}(\gamma)(t) \mp \frac{m_{k-1}(t)}{\sqrt{m_{k-1}^{2}(t)+n_{k-1}^{2}(t)}} \nu_{k-1}(t)
$$

Proof. By Proposition 4.8, the case of $k=1$ holds.

Suppose that the case of $k$ holds. We consider $\mathcal{E} v\left(\mathcal{E} v^{k}(\gamma)\right)$. By the assumption, $\left(\mathcal{E} v^{k}(\gamma), \nu_{k}\right)$ is a Legendre immersion with the curvature $\left(m_{k}, n_{k}\right)$. By Proposition 4.8, the $(k+1)$-th evolute of the front is given by

$$
\mathcal{E} v^{k+1}(\gamma)(t)= \pm \frac{n_{k}(t)}{\sqrt{m_{k}^{2}(t)+n_{k}^{2}(t)}} \mathcal{E} v^{k}(\gamma)(t) \mp \frac{m_{k}(t)}{\sqrt{m_{k}^{2}(t)+n_{k}^{2}(t)}} \nu_{k}(t) .
$$

Since

$$
\begin{aligned}
\frac{d}{d t} \mathcal{E} v^{k+1}(\gamma)(t)= & \pm \frac{d}{d t}\left(\frac{n_{k}(t)}{\sqrt{m_{k}^{2}(t)+n_{k}^{2}(t)}}\right) \mathcal{E} v^{k}(\gamma)(t) \pm \frac{n_{k}(t)}{\sqrt{m_{k}^{2}(t)+n_{k}^{2}(t)}} m_{k}(t) \boldsymbol{\mu}_{k}(t) \\
& \mp \frac{d}{d t}\left(\frac{m_{k}(t)}{\sqrt{m_{k}^{2}(t)+n_{k}^{2}(t)}}\right) \nu_{k}(t) \mp \frac{m_{k}(t)}{\sqrt{m_{k}^{2}(t)+n_{k}^{2}(t)} n_{k}(t) \boldsymbol{\mu}_{k}(t)} \\
= & \pm \frac{d}{d t}\left(\frac{n_{k}(t)}{\sqrt{m_{k}^{2}(t)+n_{k}^{2}(t)}}\right) \mathcal{E} v^{k}(\gamma)(t) \mp \frac{d}{d t}\left(\frac{m_{k}(t)}{\sqrt{m_{k}^{2}(t)+n_{k}^{2}(t)}}\right) \nu_{k}(t),
\end{aligned}
$$

and $\nu_{k+1}(t)=\boldsymbol{\mu}_{k}(t)=\mathcal{E} v^{k}(\gamma)(t) \times \nu_{k}(t)$, we have $\mathcal{E} v^{k+1}(\gamma)(t) \cdot \nu_{k+1}(t)=0$ and $\dot{\mathcal{E}}^{k+1}(\gamma)(t)$. $\nu_{k+1}(t)=0$. It follows that $\left(\mathcal{E} v^{k+1}(\gamma), \nu_{k+1}\right): I \rightarrow \Delta \subset S^{2} \times S^{2}$ is a Legendre curve. By 
definition, we have $\boldsymbol{\mu}_{k+1}(t)=\mathcal{E} v^{k+1}(\gamma)(t) \times \nu_{k+1}(t)$ and

$$
\begin{aligned}
m_{k+1}(t)= & \frac{d}{d t} \mathcal{E} v^{k+1}(\gamma)(t) \cdot \boldsymbol{\mu}_{k+1}(t) \\
= & -\frac{d}{d t}\left(\frac{n_{k}(t)}{\sqrt{m_{k}^{2}(t)+n_{k}^{2}(t)}}\right) \frac{m_{k}(t)}{\sqrt{m_{k}^{2}(t)+n_{k}^{2}(t)}} \mathcal{E} v^{k}(\gamma)(t) \cdot\left(\nu_{k}(t) \times \boldsymbol{\mu}_{k}(t)\right) \\
& -\frac{d}{d t}\left(\frac{m_{k}(t)}{\sqrt{m_{k}^{2}(t)+n_{k}^{2}(t)}}\right) \frac{n_{k}(t)}{\sqrt{m_{k}^{2}(t)+n_{k}^{2}(t)}} \nu_{k}(t) \cdot\left(\mathcal{E} v^{k}(\gamma)(t) \times \boldsymbol{\mu}_{k}(t)\right) \\
= & \frac{\dot{m}_{k}(t) n_{k}(t)-m_{k}(t) \dot{n}_{k}(t)}{m_{k}^{2}(t)+n_{k}^{2}(t)} .
\end{aligned}
$$

Moreover, since $\dot{\nu}_{k+1}(t)=\dot{\boldsymbol{\mu}}_{k}(t)=-m_{k}(t) \mathcal{E} v^{k}(\gamma)(t)-n_{k}(t) \nu_{k}(t)$, we have

$$
\begin{aligned}
n_{k+1}(t)= & \dot{\nu}_{k+1}(t) \cdot \boldsymbol{\mu}_{k+1}(t) \\
= & \pm \frac{m_{k}^{2}(t)}{\sqrt{m_{k}^{2}(t)+n_{k}^{2}(t)}} \mathcal{E} v^{k}(\gamma)(t) \cdot\left(\nu_{k}(t) \times \boldsymbol{\mu}_{k}(t)\right) \\
& \mp \frac{n_{k}^{2}(t)}{\sqrt{m_{k}^{2}(t)+n_{k}^{2}(t)}} \nu_{k}(t) \cdot\left(\mathcal{E} v^{k}(\gamma)(t) \times \boldsymbol{\mu}_{k}(t)\right) \\
= & \pm \sqrt{m_{k}^{2}(t)+n_{k}^{2}(t)} .
\end{aligned}
$$

It follows from $n_{k+1}(t) \neq 0$ for all $t \in I$ that $\left(\mathcal{E} v^{k+1}(\gamma), \nu_{k+1}\right)$ is also a Legendre immersion with the curvature $\left(m_{k+1}, n_{k+1}\right)$. This completes the proof of Theorem.

\section{Evolutes of frontals in the sphere}

Let $(\gamma, \nu): I \rightarrow \Delta \subset S^{2} \times S^{2}$ be a Legendre curve with the curvature $(m, n)$. We define an evolute of the frontal as follows.

Definition 5.1 The evolute $\mathcal{E} v(\gamma): I \rightarrow S^{2}$ of the frontal $\gamma$ is given by

$$
\mathcal{E} v(\gamma)(t)= \pm p(t) \gamma(t) \pm q(t) \nu(t)
$$

if there exists a smooth mapping $(p, q): I \rightarrow S^{1}$ such that

$$
m(t) p(t)+n(t) q(t)=0
$$

for all $t \in I$. In this case, we say that the evolute $\mathcal{E} v(\gamma)$ exists.

Remark 5.2 If $m(t)=n(t)=0$ for all $t \in I$, that is, $\gamma(t)$ and $\nu(t)$ are constant vectors in $S^{2}$, then for any smooth mapping $(p, q): I \rightarrow S^{1}$ satisfies the condition $m(t) p(t)+n(t) q(t)=0$. Then the evolute exists but does not unique.

The uniqueness condition is well-known as a topological condition.

Lemma 5.3 Suppose that there exists a continuous mapping $(p, q): I \rightarrow S^{1}$ such that $p(t)=$ $n(t) / \sqrt{m^{2}(t)+n^{2}(t)}$ and $q(t)=-m(t) / \sqrt{m^{2}(t)+n^{2}(t)}$ on $X=\left\{t \in I \mid m^{2}(t)+n^{2}(t) \neq 0\right\}$. Then the mapping $(p, q)$ is a unique if and only if $X$ is a dense subset of $I$. 
Let $(\gamma, \nu): I \rightarrow \Delta \subset S^{2} \times S^{2}$ be a Legendre curve with the curvature $(m, n)$. In this section, we assume that $X=\left\{t \in I \mid m^{2}(t)+n^{2}(t) \neq 0\right\}$ is a dense subset of $I$, that is, the set of regular points of the Legendre curve $(\gamma, \nu)$ is a dense subset of $I$. This condition follows that if such a smooth mapping $(p, q): I \rightarrow S^{2}$ exists, then the uniqueness condition is satisfied by Lemma 5.3. Note that if the singular points $(\gamma, \nu)$ are isolated, then the condition that $X$ is a dense subset of $I$ is satisfied.

The existence condition of the evolute of a frontal is as follows. It is a quit different property between the evolute of a frontal in the sphere and in the Euclidean plane (cf. [8]).

Proposition 5.4 Let $(\gamma, \nu): I \rightarrow \Delta \subset S^{2} \times S^{2}$ be a Legendre curve with the curvature $(m, n)$. If $m(t)$ or $n(t)$ does not belong to $\mathfrak{m}_{1}^{\infty}$ around $t_{0}$, then the evolute of the frontal $\mathcal{E} v(\gamma)$ exists around $t_{0}$.

Proof. Suppose that $m(t) \notin \mathfrak{m}_{1}^{\infty}$ around $t_{0}$. There exists a smooth function $\lambda:\left(I, t_{0}\right) \rightarrow \mathbb{R}$ such that $n(t)=\lambda(t) m(t)$ around $t_{0}$. We put

$$
p(t)=\frac{\lambda(t)}{\sqrt{\lambda(t)^{2}+1}}, q(t)=-\frac{1}{\sqrt{\lambda(t)^{2}+1}} .
$$

Then the condition $m(t) p(t)+n(t) q(t)=0$ holds around $t_{0}$. Therefore the evolute of the frontal $\mathcal{E} v(\gamma)$ exists around $t_{0}$. By the similar arguments, we can prove the case of $n(t) \notin \mathfrak{m}_{1}^{\infty}$ around $t_{0}$.

Proposition 5.5 Let $(\gamma, \nu): I \rightarrow \Delta \subset S^{2} \times S^{2}$ be a Legendre curve with the curvature $(m, n)$. If the evolute $\mathcal{E} v(\gamma)$ of the frontal exists with $(p, q): I \rightarrow S^{1}$ satisfies (5), then the evolute $\mathcal{E} v(\gamma)$ is also a frontal. More precisely, $(\mathcal{E} v(\gamma), \boldsymbol{\mu}): I \rightarrow \Delta \subset S^{2} \times S^{2}$ is a Legendre curve with the curvature

$$
m_{\mathcal{E} v}(t)=\dot{p}(t) q(t)-p(t) \dot{q}(t), n_{\mathcal{E} v}(t)=\mp m(t) q(t) \pm n(t) p(t) .
$$

Proof. By the Frenet Serret type formula (Proposition 2.2), we have

$$
\begin{aligned}
\dot{\mathcal{E}} v(\gamma)(t) & = \pm \dot{p}(t) \gamma(t) \pm p(t) \dot{\gamma}(t) \pm \dot{q}(t) \nu(t) \pm q(t) \dot{\nu}(t) \\
& = \pm \dot{p}(t) \gamma(t) \pm \dot{q}(t) \nu(t) \pm(m(t) p(t)+n(t) q(t)) \boldsymbol{\mu}(t) \\
& = \pm \dot{p}(t) \gamma(t) \pm \dot{q}(t) \nu(t) .
\end{aligned}
$$

By definition, $\boldsymbol{\mu}(t)=\gamma(t) \times \nu(t)$. Then $(\mathcal{E} v(\gamma), \boldsymbol{\mu})$ is a Legendre curve. We denote $\boldsymbol{\mu}_{\mathcal{E} v}(t)=$ $\mathcal{E} v(\gamma)(t) \times \boldsymbol{\mu}(t)= \pm q(t) \gamma(t) \mp p(t) \nu(t)$. Thus, the curvature is given by

$$
\begin{aligned}
m_{\mathcal{E} v}(t) & =\dot{\mathcal{E} v}(\gamma)(t) \cdot \boldsymbol{\mu}_{\mathcal{E} v}(t)=\dot{p}(t) q(t)-p(t) \dot{q}(t), \\
n_{\mathcal{E} v}(t) & =\dot{\boldsymbol{\mu}}(t) \cdot \boldsymbol{\mu}_{\mathcal{E} v}(t)=\mp m(t) q(t) \pm n(t) p(t) .
\end{aligned}
$$

Remark 5.6 By Proposition 5.5, if $n_{\mathcal{E} v}(t)=0$, then we have $m(t)=n(t)=0$. Hence if the set of regular points of the Legendre curve $(\gamma, \nu)$ is a dense subset of $I$, then the set of regular points of $(\mathcal{E} v(\gamma), \boldsymbol{\mu})$ is also a dense subset of $I$. By Proposition 5.4, if $m_{\mathcal{E} v}(t)$ or $n_{\mathcal{E} v}(t)$ dose not belong to $\mathfrak{m}_{1}^{\infty}$, then there exists unique the second evolute $\mathcal{E} v^{2}(\gamma)$ of the Legendre curve $(\gamma, \nu)$ at least locally. 


\section{$6 \quad$ Examples}

We give examples of the evolutes of fronts and frontals.

Example 6.1 (Spherical nephroid) Let $(\gamma, \nu):[0,2 \pi) \rightarrow \Delta \subset S^{2} \times S^{2}$ be

$$
\begin{aligned}
\gamma(t) & =\left(\frac{3}{4} \cos t-\frac{1}{4} \cos 3 t, \frac{3}{4} \sin t-\frac{1}{4} \sin 3 t, \frac{\sqrt{3}}{2} \cos t\right), \\
\nu(t) & =\left(\frac{3}{4} \sin t-\frac{1}{4} \sin 3 t,-\frac{3}{4} \cos t-\frac{1}{4} \cos 3 t,-\frac{\sqrt{3}}{2} \sin t\right) .
\end{aligned}
$$

Since

$$
\dot{\gamma}(t)=\left(-\frac{3}{4} \sin t+\frac{3}{4} \sin 3 t, \frac{3}{4} \cos t-\frac{3}{4} \cos 3 t,-\frac{\sqrt{3}}{2} \sin t\right),
$$

we have $\gamma(t) \cdot \nu(t)=0$ and $\dot{\gamma}(t) \cdot \nu(t)=0$. Hence $(\gamma, \nu):[0,2 \pi) \rightarrow \Delta \subset S^{2} \times S^{2}$ is a Legendre curve. By definition, we have

$$
\boldsymbol{\mu}(t)=\left(\frac{\sqrt{3}}{2} \cos 2 t, \frac{\sqrt{3}}{2} \sin 2 t,-\frac{1}{2}\right),
$$

and the curvature $(m(t), n(t))=(\sqrt{3} \sin t, \sqrt{3} \cos t)$. It follows that $(\gamma, \nu)$ is a Legendre immersion. The evolute of the front is given by

$$
\begin{aligned}
\mathcal{E} v(\gamma)(t) & = \pm \frac{n(t)}{\sqrt{m^{2}(t)+n^{2}(t)}} \gamma(t) \mp \frac{m(t)}{\sqrt{m^{2}(t)+n^{2}(t)}} \nu(t) \\
& = \pm \cos t \gamma(t) \mp \sin t \nu(t) \\
& = \pm\left(\frac{1}{2} \cos 2 t, \frac{1}{2} \sin 2 t, \frac{\sqrt{3}}{2}\right)
\end{aligned}
$$

and the curvature of $(\mathcal{E} v(\gamma), \boldsymbol{\mu})$ is $\left(m_{\mathcal{E} v}(t), n_{\mathcal{E} v}(t)\right)=(1, \pm \sqrt{3})$ by Proposition 4.8. Then the second evolute of the front is given by

$$
\mathcal{E} v^{2}(\gamma)(t)= \pm \frac{n_{\mathcal{E} v}(t)}{\sqrt{m_{\mathcal{E} v}^{2}(t)+n_{\mathcal{E} v}^{2}(t)}} \mathcal{E} v(t) \mp \frac{m_{\mathcal{E} v}(t)}{\sqrt{m_{\mathcal{E} v}^{2}(t)+n_{\mathcal{E} v}^{2}(t)}} \boldsymbol{\mu}(t)= \pm(0,0,1)
$$

Example 6.2 Let $n, m$ and $k$ be natural numbers with $m=k+n$. Consider a Legendre curve $(\gamma, \nu): \mathbb{R} \rightarrow \Delta \subset S^{2} \times S^{2}$ by

$$
\gamma(t)=\frac{1}{\sqrt{1+t^{2 n}+t^{2 m}}}\left(1, t^{n}, t^{m}\right), \nu(t)=\frac{1}{\sqrt{n^{2}+m^{2} t^{2 k}+k^{2} t^{2 m}}}\left(k t^{m},-m t^{k}, n\right),
$$

see Example 2.8. Then the curvature is given by

$$
m(t)=\frac{-t^{n-1} \sqrt{n^{2}+m^{2} t^{2 k}+k^{2} t^{2 m}}}{1+t^{2 n}+t^{2 m}}, n(t)=\frac{k n m t^{k-1} \sqrt{1+t^{2 n}+t^{2 m}}}{n^{2}+m^{2} t^{2 k}+k^{2} t^{2 m}} .
$$


Note that $(\gamma, \nu)$ is a Legendre immersion when $k=1$ or $n=1$. We put $1<n \leq k=n+r$ for some natural number $r$. Then the evolute of the frontal is given by

$$
\mathcal{E} v(\gamma)(t)= \pm p(t) \gamma(t) \pm q(t) \nu(t)
$$

where

$$
\begin{aligned}
& p(t)=\frac{\left(1+t^{2 n}+t^{2 m}\right)^{\frac{3}{2}} k n m t^{r}}{\sqrt{\left(1+t^{2 n}+t^{2 m}\right)^{3}(k n m)^{2} t^{2 r}+\left(n^{2}+m^{2} t^{2 k}+k^{2} t^{2 m}\right)^{3}}}, \\
& q(t)=\frac{\left(n^{2}+m^{2} t^{2 k}+k^{2} t^{2 m}\right)^{\frac{3}{2}}}{\sqrt{\left(1+t^{2 n}+t^{2 m}\right)^{3}(k n m)^{2} t^{2 r}+\left(n^{2}+m^{2} t^{2 k}+k^{2} t^{2 m}\right)^{3}}} .
\end{aligned}
$$

For example, when $n=2, m=5, k=3$ and $r=1$, then we have

$$
\gamma(t)=\frac{1}{\sqrt{1+t^{4}+t^{10}}}\left(1, t^{2}, t^{5}\right), \nu(t)=\frac{1}{\sqrt{4+25 t^{6}+9 t^{10}}}\left(3 t^{5},-5 t^{3}, 2\right)
$$

and

$$
\begin{aligned}
& p(t)=\frac{30 t\left(1+t^{4}+t^{10}\right)^{\frac{3}{2}}}{\sqrt{30^{2} t^{2}\left(1+t^{4}+t^{10}\right)^{3}+\left(4+25 t^{6}+9 t^{10}\right)^{3}}} \\
& q(t)=\frac{\left(4+25 t^{6}+9 t^{10}\right)^{\frac{3}{2}}}{\sqrt{30^{2} t^{2}\left(1+t^{4}+t^{10}\right)^{3}+\left(4+25 t^{6}+9 t^{10}\right)^{3}}} .
\end{aligned}
$$

Then the evolute of the frontal is given by

$$
\mathcal{E} v(\gamma)(t)= \pm \frac{30 t\left(1+t^{4}+t^{10}\right)\left(1, t^{2}, t^{5}\right)+\left(4+25 t^{6}+9 t^{10}\right)\left(3 t^{5}-5 t^{3}, 2\right)}{\sqrt{30^{2} t^{2}\left(1+t^{4}+t^{10}\right)^{3}+\left(4+25 t^{6}+9 t^{10}\right)^{3}}}
$$

For a smooth curve $\gamma$ on $S^{2}$, we say that $t$ is an ordinary rhamphoid cusp if $\dot{\gamma}(t)=0, \ddot{\gamma}(t) \neq$ $0, \gamma^{(3)}(t)=3 \lambda \ddot{\gamma}(t)$ for some $\lambda \in \mathbb{R}$, and $\gamma^{(5)}(t)$ is linearly independent of $\ddot{\gamma}(t)$ and $\gamma^{(4)}(t)$ (cf. $[16,17])$. Therefore, this is an example that 0 is an ordinary rhamphoid cusp of $\gamma$, but 0 is not of $\nu$ (cf. Proposition 2 in [16] page 219).

\section{References}

[1] V. I. Arnol'd, Singularities of Caustics and Wave Fronts, Mathematics and Its Applications 62 Kluwer Academic Publishers, 1990.

[2] V. I. Arnol'd, The geometry of spherical curves and quaternion algebra, Russian Math. Surveys, 50 (1995), 1-68

[3] V. I. Arnol'd, S. M. Gusein-Zade and A. N. Varchenko, Singularities of Differentiable Maps vol. I, Birkhäuser, 1986.

[4] R. L. Bishop, There is more than one way to frame a curve. Amer. Math. Monthly, 82 (1975), $246-251$.

[5] J. W. Bruce and P. J. Giblin, Curves and singularities. A geometrical introduction to singularity theory. Second edition, Cambridge University Press, Cambridge, 1992. 
[6] T. Fukunaga and M. Takahashi, Existence and uniqueness for Legendre curves, J. Geometry. 104 (2013), 297-307.

[7] T. Fukunaga and M. Takahashi, Evolutes of fronts in the Euclidean plane, J. Singularity. 10 (2014), 92-107.

[8] T. Fukunaga and M. Takahashi, Evolutes and involutes of frontals in the Euclidean plane, Demonstratio Math. 48 (2015), 147-166.

[9] C. G. Gibson, Singular points of smooth mappings, Research Notes in Mathematics, 25, Pitman (Advanced Publishing Program), Boston, Mass.-London, 1979.

[10] C. G. Gibson, Elementary geometry of differentiable curves. An undergraduate introduction, Cambridge University Press, Cambridge, 2001.

[11] A. Gray, E. Abbena, and S. Salamon, Modern differential geometry of curves and surfaces with Mathematica. Third edition, Studies in Advanced Mathematics. Chapman and Hall/CRC, Boca Raton, FL, 2006.

[12] S. Honda and M. Takahashi, Framed curves in the Euclidean space, to appear in Advances geometry (2015).

[13] T. Nagai, The Gauss map of a hypersurface in Euclidean sphere and the spherical Legendrian duality. Topology Appl. 159 (2012), 545-554.

[14] T. Nishimura, Normal forms for singularities of pedal curves produced by non-singular dual curve germs in $S^{n}$. Geom. Dedicata. 133 (2008), 59-66.

[15] T. Nishimura, Singularities of pedal curves produced by singular dual curve germs in $S^{n}$. Demonstratio Math. 43 (2010), 447-459.

[16] I. R. Porteous, Some remarks on duality in $S^{3}$, Geometry and topology of caustics-CAUSTICS '98, Banach Center Publ., 50 (1999), 217-226.

[17] I. R. Porteous, Geometric differentiation. For the intelligence of curves and surfaces. Second edition. Cambridge University Press, Cambridge, 2001.

[18] M. C. Romero Fuster, Stereographic projections and geometric singularities. Workshop on Real and Complex Singularities (So Carlos, 1996). Mat. Contemp. 12 (1997), 167-—182.

[19] R. Uribe-Vargas, On polar duality, Lagrange and Legendre singularities and stereographic projection to quadrics. Proc. London Math. Soc. 87 (2003), 701-—724.

[20] R. Uribe-Vargas, Rigid body motions and Arnold's theory of fronts on $S^{2} \subset \mathbb{R}^{3}$, J. Geom. Phys. 45 (2003), 91-104.

[21] R. Uribe-Vargas, Theory of fronts on the 2-sphere and the theory of space curves, J. Math. Sci. (N.Y.) 126 (2005), 1344-1353.

[22] V. M. Zakalyukin, Reconstructions of fronts and caustics depending on a parameter and versality of mappings. J. Soviet Math. 27 (1983), 2713-2735.

Masatomo Takahashi,

Muroran Institute of Technology, Muroran 050-8585, Japan,

E-mail address: masatomo@mmm.muroran-it.ac.jp 